\title{
Karate Kumite: How to Optimize Performance - Análisis de libro
}

\author{
Tomás HERRERA-VALENZUELA ${ }^{1,2^{*}}$ \& Pablo VALDÉS-BADILLA ${ }^{3,4,5}$
}

\author{
${ }^{1}$ Laboratorio de Inmunología de la Reproducción, Facultad de Química y Biología. Universidad de \\ Santiago de Chile, USACH (Chile)
}

2 Grupo de Investigación en Deportes de Combate y Artes Marciales, GIDECAM. Laboratorio de Ciencias de la Actividad Física, el Deporte y la Salud. Facultad de Ciencias Médicas. Universidad de Santiago de Chile, USACH (Chile)

${ }^{3}$ Instituto de Actividad Física y Salud, Universidad Autónoma de Chile (Chile).

${ }^{4}$ Pedagogía en Educación Física, Facultad de Educación, Universidad Autónoma de Chile, sede Temuco (Chile).

${ }^{5}$ Programa de Doctorado en Ciencias de la Actividad Física, Facultad de Ciencias de la Educación, Universidad Católica del Maule (Chile).

Recepción: 11/08/2016; Aceptación: 27/12/2016; Publicación: 27/12/2016.

\begin{abstract}
Resumen
En este trabajo se analiza el libro Karate Kumite: How to Optimize Performance, editado en inglés por el Dr. Helmi Chaabane en el año 2015. La obra se estructura en cinco capítulos y participan en ella un total de siete autores. El primer capítulo, "Performance Analysis in Karate", analiza la estructura temporal del combate. El segundo capítulo, "Physiological Characteristics of Karate Athletes and Karate-Specific Tasks", describe el costo y contribución de los sistemas energéticos, la respuesta cardiovascular, del lactato sanguíneo y hormonal frente al combate. El tercer capítulo "Physical Determinants of Karate Kumite" estudia la potencia muscular, fuerza máxima, aceleración, velocidad, tiempo de reacción y flexibilidad del karate. El cuarto capítulo, "Training Load Monitoring", describe distintos métodos para la monitorización de la carga interna. En el último capítulo, "Physical and Physiological Assessment", presenta test generales y específicos utilizados en el karate. El amplio currículo investigador de los autores, así como la extensa utilización de literatura actualizada como base del libro, dan como resultado una obra de alto valor científico que, a la vez, puede ser consultada sin restricciones ya que se ha editado en formato digital y en acceso libre. En conclusión, se trata de una obra muy recomendable para entrenadores, practicantes y profesionales relacionados con el karate.

Palabras clave: artes marciales; deportes de combate; karate; redimiento deportivo; entrenamiento.
\end{abstract}

\section{Karate Kumite: How to Optimize Performance - Book analysis}

\begin{abstract}
This work reviews the book entitled Karate Kumite: How to Optimize Performance, edited in English by Helmi Chaabane, PhD., in 2015. The book is composed of five chapters and a total of seven authors participate in it. The first chapter, "Performance Analysis in Karate", analyzes the temporal structure of combat. The second chapter, "Physiological Characteristics of Karate Athletes and Karate-Specific Tasks" describes the cost and contribution of energy systems, cardiovascular response, blood lactate and hormonal response to combat. The third chapter, "Physical Determinants of Karate Kumite", analyzes the muscular power, maximum strength, acceleration, speed, reaction time and flexibility of karate. The fourth chapter, "Training Load Monitoring", describes different methods for monitoring the internal load. Finally the last chapter,
\end{abstract}

\section{Karate Kumite: How to Optimize Performance - Análise do livro}

\section{Resumo}

Este trabalho analisa o livro Karate Kumite: How to Optimize Performance, editado em inglês por Helmi Chaabane, em 2015. A obra estrutura-se em cinco capítulos e nela participam sete autores. 0 primeiro capítulo, "Performance Analysis in Karate", analisa a estrutura temporal do combate. 0 segundo capítulo, "Physiological Characteristics of Karate Athletes and Karate-Specific Tasks", descreve o custo e a contribuição dos sistemas energéticos, a resposta cardiovascular, o lactato sanguíneo e hormonal frente ao combate. 0 terceiro capítulo, "Physical Determinants of Karate Kumite", estuda a potência muscular, força muscular, aceleração, velocidade, tempo de reação e flexibilidade do karaté. O quarto capítulo, "Training Load Monitoring", descreve distintos métodos para a monitorização da carga interna. 0 último capítulo, "Physical and Physiological Assessment", 
"Physical and Physiological Assessment", presents general and specific tests used in karate. The sound research background of the authors, as well as the extensive use of up to date literature, results in a book of high scientific value. In addition, this is a digital, open-access book that can be accessed without restriction. In conclusion, this is a highly recommended book for coaches, practitioners and professionals related to karate.

Keywords: martial arts; combat sports; karate; sports performance; training. apresenta testes gerais e específicos utilizados no karaté. 0 amplo currículo dos investigadores e/ou autores, assim como a extensa utilização da literatura atualizada como base no livro, dão como resultado uma obra de alto valor científico, que pode ser consultada sem restrições, dado que foi editada em formato digital e de livre acesso. Em conclusão, trata-se de uma obra recomendável para os treinadores, praticantes e profissionais relacionados com o karaté.

Palavras-chave: artes marciais; desportos de combate; karaté; rendimento desportivo; treino.

\section{Introducción}

Existe numerosa literatura sobre las artes marciales y los deportes de combate (véanse e.g., Gutiérrez-García \& Pérez-Gutiérrez, 2009; Pérez-Gutiérrez, Brown, Álvarez, \& Gutiérrez-García, 2014; Pérez-Gutiérrez \& Gutiérrez-García, 2009; Pérez-Gutiérrez, Valdés-Badilla, Gómez-Alonso, Gutiérrez-García, 2015; Peset, et al. 2013). A modo de ejemplo, Pérez-Gutiérrez y Gutiérrez-García (2008), recogieron en su estudio bibliométrico un total de 1.285 libros publicados en España durante el período 1906-2006, de los cuales 309 correspondían a libros sobre karate. Sin embargo, es escasa la literatura científica relativa al karate que contemple aspectos fisiológicos y de rendimiento deportivo.

\section{La obra}

Karate Kumite: How to Optimize Performance, es un libro editado por el Dr. Helmi Chaabane y publicado en formato digital abierto por Omics group (el libro es totalmente accesible desde http://www.esciencecentral.org/ebooks/karate-kumite-how-to-optimize-performance). Presenta cinco capítulos y participan en él un total de siete autores. Desde el punto de vista institucional, se observa una clara colaboración entre la Universidad de Jendouba (Túnez) y la Universidad de São Paulo (Brasil), representada por los autores Helmi Chaabane y Emerson Franchini, ambos con una alta productividad científica de trabajos relacionados con los aspectos fisiológicos de las artes marciales y deportes de combate, lo que constituye un sello de calidad.

En cuanto a la temática de los capítulos, el primero se titula "Performance Analysis in Karate" y está firmado por Emerson Franchini, Irineu Loturco y Fábio Yuzo Nakamura. En él se presenta la estructura temporal del combate simulado y de la competencia oficial en karate, exponiendo la proporción de las técnicas de ataques, contra-ataques y combinación de ataques, además de la duración del combate, el tiempo de las fases de lucha, preparación y detención. Así mismo, se presentan las diferencias entre ganadores y perdedores por categorías de peso corporal y sexo. Finalmente, se detallan algunas conclusiones y aplicaciones prácticas.

El segundo capítulo, escrito por Emerson Franchini, Ibrahim Ouergui, y Helmi Chaabene, se titula "Physiological Characteristics of Karate Athletes and Karate-Specific Tasks". En él se describe el perfil fisiológico aeróbico a través de valores referenciales de V02máx y el anaeróbico por medio de las pruebas "Wingate" y "máximo déficit acumulado de oxígeno" (MAOD). Se expone la respuesta fisiológica durante tareas específicas de karate, presentando el costo y la contribución de los sistemas energéticos durante el karate kumite. Se exhibe, a la vez, la respuesta cardiovascular de los karatekas en competencia oficial y simulada a través de la frecuencia cardíaca promedio y peak (pico). En suma a lo anterior, se aprecian registros de lactato sanguíneo antes y después de la competencia, así como las diferencias entre sexo y durante tres combates sucesivos, además de valores hormonales de testosterona y cortisol medidos en saliva. Finalmente, se presentan conclusiones y futuras directrices. Dicho capítulo cuenta con siete gráficos de barra y una tabla, facilitando la comprensión de la información.

El tercer capítulo se titula "Physical Determinants of Karate Kumite" y está firmado por Ibrahim Ouergui, Emerson Franchini y Helmi Chaabene. En esta sección se describen las características físicas del karate kumite y su relación con el rendimiento deportivo a través del 
somatotipo, porcentaje de grasa, potencia muscular, fuerza máxima, aceleración, velocidad, tiempo de reacción y flexibilidad de distintos grupos de atletas elite de la especialidad. El capítulo finaliza con las consabidas conclusiones y aplicaciones prácticas.

El cuarto capítulo se titula "Training Load Monitoring", y fue escrito por Nizar Houcine, Ibrahim Ouergui y Helmi Chaabene. En este apartado se analizan diferentes métodos de cuantificación de la carga de trabajo, entre ellos: a) sistemas basados en la frecuencia cardíaca en base al impulso de entrenamiento (TRIMP) propuesto por distintos autores (Banister, Morton, Lucia, Edwards, y Stagno), b) el método TRIMP en base al lactato sanguíneo, c) el método de percepción del esfuerzo en base a la escala de Borg y la versión modificada. Posteriormente, se presentan datos de monitoreo de la carga durante el entrenamiento y competencia de karate, junto a la comparación entre los métodos.

El último capítulo, escrito por Helmi Chaabene y Yassine Negra, se titula "Physical and Physiological Assessment". En él se exponen los principales factores a considerar al momento de elegir un test, explicando la importancia de la validez, confiabilidad y sensibilidad de dichas mediciones. Se analizan pruebas de rendimiento físico relacionadas con la fuerza máxima, potencia muscular y evaluaciones fisiológicas generales (tanto aeróbicas como anaeróbicas). Luego, se presentan pruebas específicas, como el Aerobic Karate Test, el Special Karate Fitness Test y el Karate Specific Agility Test. Para finalizar con las conclusiones que fundamentan la importancia de las evaluaciones en karate.

Como puntos fuertes del libro, destacan la actualidad de las referencias utilizadas, que permiten obtener en un solo texto mucha de la actualidad científica relacionada con el karate. Igualmente, la solvencia de los autores otorga confiabilidad y rigor al texto, debido al importante volumen de investigaciones con las que cuenta cada uno sobre la temática. Las secuencias de tablas e imágenes que acompañan al texto principal permiten visualizar con claridad y de manera didáctica la información presentada. A pesar de no encontrar puntos débiles importantes, una segunda edición podría incluir otras variables que intervienen en el rendimiento deportivo, como por ejemplo la psicología, nutrición y el control del peso, además de incorporar el análisis de la nueva distribución para las categorías olímpicas en kumite y kata.

\section{3.- Conclusión}

El alto valor científico del texto reside principalmente en la calidad de los autores, quienes ostentan una elevada reputación internacional y son académicos dedicados a la investigación sobre las artes marciales y deportes de combate, pertenecientes a prestigiosas universidades y centros de investigación. Además, el libro cuenta con una rigurosa revisión de la literatura existente, alcanzando un total de 147 referencias, en su mayoría artículos de investigaciones publicados en los últimos 10 años. Esto permitirá a entrenadores y practicantes de karate encontrar en un solo libro una alta cantidad de información científica sintetizada y analizada, además de recomendaciones para su aplicación práctica, lo cual, se complementa con la incorporación de tablas e imágenes que resumen de forma clara y sencilla la información. Otros elementos a destacar es que la obra es digital y de libre acceso, y que permite a través de su sitio web utilizar la herramienta Google traductor para poder facilitar su lectura en diferentes idiomas.

Considerando la escasez de este tipo de textos, Karate Kumite podría convertirse en un libro de consulta para entrenadores, profesores, instructores y estudiantes de otras artes marciales y deportes de combate. Recomendamos a todo entrenador de karate interesado en el rendimiento deportivo revisar este valioso libro, dado a ser un aporte para la fundamentación teórica y práctica tanto del entrenamiento como de la competición de karate kumite.

\section{Referencias}

Chaabene, H. (Ed.). (2015). Karate Kumite: How to Optimize Performance. Physical Determinants of Karate Kumite. Foster City, CA: OMICS. Recuperado de http://www.esciencecentral.org/ebooks/karate-kumite-how-to-optimize-performance 
Gutiérrez-García, C., \& Pérez-Gutiérrez, M. (2009). Study on scientific production in martial arts in Spain from 1990 to present. En W. J. Cynarski (Ed.), Martial Arts and Combat Sports Humanistic Outlook (pp. 90-115). Rzeszow: Wydawnictwo Uniwersytetu Rzeszowskiego.

Pérez-Gutiérrez, M., Brown, D.H.K., Álvarez, E., \& Gutiérrez-García, C. (2014). Asian martial arts bibliographies (1943-2013): a Review. IDO movement for culture. Journal of Martial Arts Anthropology, 14(3), 1-9.

Pérez-Gutiérrez, M. \& Gutiérrez-García, C. (2008). Estudio bibliométrico: sobre las monografías de artes marciales publicadas en España (1906-2006). Revista de Artes Marciales Asiáticas, 3(4), 22-3.

Pérez-Gutiérrez, M., \& Gutiérrez-García, C. (2009). Bibliometric analysis of karate monographs in Spain (1963-2006). En W. J. Cynarski (Ed.), Martial Arts and Combat Sports - Humanistic Outlook (pp. 116-126). Rzeszow: Wydawnictwo Universytetu Rzeszowskiego.

Pérez-Gutiérrez, M., Valdés-Badilla, P., Gómez-Alonso, M.T., \& Gutiérrez-García, C. (2015). Bibliometric analysis of taekwondo articles published in the Web of Science (1989-2013). IDO Movement for Culture. Journal of Martial Arts Anthropology, 15(3), 8-21.

Peset, F., Ferrer-Sapena, A., Villamón, M., González, L.M., Toca-Herrera, J.L., \& Aleixandre-Benavent, R. (2013). Scientific literature analysis of Judo in Web of Science. Archives of Budo, 9(2), 81-9.

\section{Author's biographical data}

Tomás Herrera Valenzuela, es Profesor de Educación Física, y se encuentra realizando un Postdoctorado en la Facultad de Química y Biología de la Universidad de Santiago de Chile, USACH, Chile. Adicionalmente es coordinador de investigación de la Escuela de Ciencias de la Actividad Física, el Deporte y la Salud de la misma Universidad, donde imparte tres asignaturas (Fisiología del ejercicio; metodología de la investigación aplicada; deportes de combate), mientras que su principal línea de investigación es la Fisiología aplicada a las AM\&DC. En relación a las AM\&DC, ha sido técnico nacional de Taekwondo y presenta el grado de $4^{\circ}$ dan en la especialidad. E-mail: tomas.herrera@usach.cl

Pablo Valdés Badilla, es Profesor de Educación Física, y se encuentra cursando el Programa de Doctorado en Ciencias de la Actividad Física de la Universidad Católica del Maule, Chile. Actualmente se desempeña como Administrador-Docente del Centro de Deportes y Salud, como Docente-Investigador del Instituto de Actividad Física y Salud, y como Profesor de la carrera de Pedagogía en Educación Física, todos dependientes de la Universidad Autónoma de Chile, sede Temuco, Chile. Sus principales líneas de investigación son la Actividad Física y la Salud, y las AM\&DC, contando con diversas publicaciones indexadas. En relación a las AM\&DC, ha sido técnico nacional de Taekwondo y presenta el grado de 5o dan en la especialidad. E-mail: pablo.valdes@uautonoma.cl 